\title{
Dual-mode output in half open cavity random fibre laser
}

\begin{abstract}
The mechanism of laser mode in fibre laser has been a popular talking issue since decades ago. Numerous mode mechanisms are revealed from time to time such as LP and TM. From these modes, fundamental mode and higher-order mode exhibit practical applications such as in fibre laser. Based on this perspective, we employ the mode mechanism in random fibre laser with half open cavity that generates dual-mode output which consists of LP01 and LP11 modes. The investigation on our proposed laser setup is beneficial for developing photonics system that involves the implementation of few-modes mechanism.
\end{abstract}

Keyword: Random fibre laser; Dual-mode laser; Fibre laser; Few-mode fibre; Space division multiplexing 